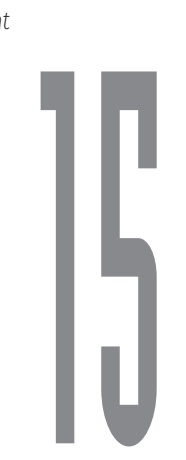

\title{
TRANSFERENCIA TECNOLÓGICA EN PROGRAMAS PÚBLICOS DE COOPERACIÓN UNIVERSIDAD-EMPRESA. PROPUESTA DE UN MODELO BASADO EN EVIDENCIA EMPÍRICA
}

\author{
JOSÉ ALBORS GARRIGÓS' \\ ANTONIO HIDALGO NUCHERA ${ }^{2}$ \\ ' DEPARTAMENTO DE ORGANIZACIÓN DE EMPRESAS. ESCUELATÉCNICA SUPERIOR DE INGENIEROS INDUSTRIALES \\ UNIVERSIDAD POLITÉCNICA DEVALENCIA \\ 2 DEPARTAMENTO DE INGENIERÍA DE ORGANIZACIÓN. ESCUELA TÉCNICA SUPERIOR DE INGENIEROS INDUSTRIALES \\ UNIVERSIDAD POLITÉCNICA DE MADRID
}

\begin{abstract}
Resumen: Los retos de las políticas públicas de innovación están relacionados con la mejora de la transferencia del conocimiento y la tecnología desde la universidad y los centros de investigación a la industria, de ahí la nueva orientación de las políticas tecnológicas nacionales y de la Unión Europea. El objetivo de este trabajo es contribuir a la comprensión de los factores subyacentes al proceso de transferencia de tecnología de la universidad a la industria. En el mismo se intenta proporcionar respuestas a un número de preguntas relacionadas con los factores de éxito y las barreras que obstaculizan la transferencia de tecnología y su impacto final en la industria.
\end{abstract}

Palabras clave: Transferencia de tecnología, cooperación universidad-empresa.

\section{Agradecimientos}

Los autores agradecen la ayuda financiera recibida del Ministerio de Educación y Ciencia a través del proyecto DPI2005-24328-E del Plan Nacional de $1+D+1$.

\section{Introducción}

Uno de los problemas reconocidos dentro de la gestión de la innovación y la tecnología en España es el relacionado con la transferencia de tecnología y conocimiento entre universidad y empresa. Los retos de las políticas públicas de innovación están relacionados con la mejora de la transferencia del conocimiento y la tecnología desde la universidad y los centros de investigación a la industria (Schmiemann y Durvy, 2003). El objetivo de este trabajo es contribuir a la comprensión de los factores subyacentes al proceso de transferencia de tecnología de la universidad a la industria.

La presentación de este trabajo se ha organizado de acuerdo a la siguiente estructura. En primer lugar, se realiza un análisis de la literatura académica en lo referente a la transferencia de tecnología desde la universidad a la empresa. A continuación, se analizan los objetivos y enfoques del trabajo con más detalle y se describe la metodología y los resultados obtenidos. El trabajo finaliza con la exposición de las conclusiones.

\section{Estado del arte.Transferencia de tecnología universidad-empresa}

La transferencia de tecnología se ha definido como una «interacción intencional, orientada hacia un objetivo, entre dos o más entidades sociales, durante las cuales el stock de conocimiento tecnológico permanece estable o aumenta por medio de la transfe- 
rencia de unos o más componentes de la tecnología» (Autio y Laamanen, 1995). La literatura académica se ha ocupado extensamente de la transferencia de tecnología en todos los aspectos que la conciernen (Bozeman, 2000; Reisman, 2005).

Recientemente se ha subrayado el rol que esta transferencia juega en la competitividad del área de influencia geográfica de la universidad (Matkin, 1990; Ronde y Hussler, 2005; Mowery, 2005; Etzkowitz, 2005; Gunasekara, 2006; Cooke, 2006; Mazzoleni, 2006), o en la innovación tecnológica (Branscomb, 1993). También se ha subrayado el impacto que la transferencia de tecnología tiene en el propio proceso educativo (Stephan, 200I) en el rol de la cultura de la universidad ( $\bigcirc$ 'Shea et al., 2005), en la importancia de la comunicación en el proceso (Williams y Gibson, 1990), así como en factores relacionados con las relaciones, la confianza, la proximidad geográfica las redes y las relaciones informales (Harmon et al., 1997; Santoro, 200 I). También se ha analizado el tema del emprendedurismo universitario como un medio de transferencia tecnológica (Etzkowitz et al., 2000; Mowery y Shane, 2002; Etzkowitz, 2004; Siegel et al., 2004; Bercovitz y Feldmann, 2006).

En relación al ámbito de la cooperación entre agentes, Gorschek et al. (2006) proponen un modelo de transferencia de tecnología universidad-empresa basado en una agenda de investigación en la que el trabajo del investigador no es simplemente investigar, sino procurar que ocurra la transferencia de tecnología. Lane (1999) presenta un modelo conceptual del proceso, objetivos de la empresa y de la universidad (Geisler y Rubenstain, 1989; Caloghirou et al., 200 I). Se han apuntado también dos factores básicos en la discusión actual sobre transferencia tecnológica universidad-empresa (Lee, 1996; Colyvas et al., 2002): el declive de la ayuda publica en I+D, y el impacto de la cooperación universidad-empresa.

El aspecto de la propiedad industrial ha sido analizado también por numerosos autores. Así, Morgan et al. (200I) subrayan las patentes universitarias como un indicador importante de la relación universidadempresa, aunque también como una barrera a la transferencia de tecnología (Link y Scott, 200l; Thursby et al., 200 I; Shane, 2002; Meyer, 2006). La relevancia de los aspectos organizativos, las OTRIS y las barreras culturales en la transferencia de tecnología se ha subrayado también por diversos autores (Bercovitz et al., 200 I; Rogers et al., 200I; Meseri y Maital, 200 I; Siegel et al., 2003). La tecnología en si ha sido objeto de estudio desde un punto de vista contingente de su transferencia (Hannan y Freeman,
1984; Singh, 1997; Stock y Tatikonda, 2000 y 2003; Shane, 200I).

Las barreras y los facilitadores del proceso han sido ampliamente tratadas: las inherentes a la gestión de la propiedad industrial, los objetivos, la divergencia cultural y organizativa; la gestión del proyecto y como de la transferencia, la relación entre las organizaciones, la involucración de los usuarios, la capacidad tecnológica de los usuarios, el liderazgo y el impacto del conocimiento tácito (Walker y Ellis, 2000; Hall et al., 200 I; Greiner, 2003).

Los problemas asociados a las oficinas de transferencia de resultados y sus políticas han sido tratados también por diversos autores (Adams et al., 200 I; Siegel et al., 2004; Chapple et al., 2005; Anderson, 2007). Finalmente, se han analizado las políticas públicas para la promoción de la transferencia de tecnología (Brasncomb, I 993; Bozeman, 2000; Lee, 2002).

\section{Objetivos de la investigación: cuestiones y problemas a resolver}

Según el informe de la Fundación CYD (2006) sólo un 7,5\% de la I+D realizada en las universidades españolas se financia por medio de capital privado de origen empresarial frente a un 13\% en Alemania o un 30\%. El mismo informe CYD (2006) apunta que en 2004 solo el 3,5\% de las empresas innovadoras españolas (un $31,4 \%$ del conjunto de empresas españolas) cooperaron en innovación con universidades. Los datos relativos a los contratos de I+D desarrollados por la Red de Fundaciones Universidad-Empresa (3.519 contratos por valor de 73,3 millones de euros, de los que solo el $44 \%$ se relacionaban con I+D) tampoco son muy halagüeños (Fundación CYD, 2006).

El problema que se plantea en este trabajo es analizar qué variables son críticas en el proceso de transferencia tecnológica de universidad a empresa, y cuáles son las barreras y los facilitadores del proceso dentro del esquema de los proyectos de apoyo público a la investigación cooperativa.

\section{Metodología del trabajo de campo}

\section{I. Introducción}

La metodología de esta investigación se basa en una encuesta realizada a una muestra de universidades y centros de I+D y empresas participantes en dos tipos de proyectos relacionados con las tecnologías 
de robótica y producción. Por una parte proyectos de I+D de la Comisión Interministerial de Ciencia y Tecnología (CICYT). Estos proyectos son coordinados por universidades, aunque en ellos participaban empresas y centros tecnológicos. El otro grupo lo constituían los proyectos del Centro para el Desarrollo Tecnológico Industrial (CDTI). Estos proyectos estaban coordinados por empresas, aunque en ellos participaban universidades y centros tecnológicos.

El apoyo público a ambos grupos de proyectos era diferente. En el primer caso consistía en una subvención, que cubría total o parcialmente la actividad que se llevaba a cabo. En el segundo caso consistía en un crédito del 50-60\% del proyecto en condiciones especiales a interés cero. Adicionalmente podría concederse una subvención de hasta un 70\% de la contratación del centro público de investigación participante.

El trabajo analiza los resultados de la encuesta, que fue respondida por 250 organizaciones y que cubría un número de aspectos relacionados con las actitudes del participante, cuestiones relativas a la tecnología, las barreras encontradas, los mecanismos de transferencia de tecnología y el impacto final del programa.

\subsection{Población y muestra}

La base de datos utilizada ha sido la de los proyectos CICYT y los proyectos CDTI desarrollados durante el periodo 1996-2004. La composición de población y muestra ha sido la siguiente:

- Universidades (proyectos CICYT): número de proyectos 880; respuestas obtenidas 87 (9,9\%); error muestral 1,96\% con un intervalo de confianza del 95\%.

- Empresas (proyectos CDTI): número de proyectos 1303; respuestas 142 ( 10,9\%); error muestral I,73\% con intervalo de confianza del 95\%.

\subsection{Procedimiento y encuesta}

Se envió la encuesta a la población de individuos considerada (coordinadores de cada proyecto) durante Septiembre-Octubre de 2006. Simultáneamente, se efectuó un seguimiento telefónico de la misma. Igualmente se realizaron entrevistas con diversas empresas y equipos universitarios para contrastar los resultados y obtener una visión más amplia del proyecto.
A continuación se resumen las preguntas utilizadas en el estudio y que se agrupan según los epígrafes de la encuesta:

- Pregunta I. Participación, número de participantes y valoración. En esta sección se pretendía clasificar los proyectos según la modalidad y relevancia de la cooperación, así como cuantificar el nivel de la cooperación según el número de participantes. Finalmente, se pretendía obtener opiniones sobre el rol de los diversos participantes en los proyectos (universidades, centros de I+D, empresas).

- Pregunta 2. Naturaleza de las actividades. Esta pregunta se refiere al tipo de actividades que se desarrollaban en el proyecto: investigación básica, desarrollo tecnológico, ingeniería, usuario final, etc. (respuestas dicotómicas de 0 a I).

- Pregunta 3. Aspectos tecnológicos del proyecto. Aquí se trataba de obtener información sobre la tecnología y sus características en cuanto a complejidad tecnológica y organizativa, y el riesgo (escala de respuestas de I a 5).

- Pregunta 4 (a/b). Relevancia y Cumplimiento de los objetivos del proyecto. Esta pregunta hace mención a los aspectos de los diversos objetivos del proyecto, su importancia para el encuestado y grado de cumplimiento: desarrollo de nuevos productos, mejora de productos existentes, desarrollo de nuevos procesos, mejora procesos existentes, demostradores, plantas piloto, adquisición de conocimientos, mejora gestión del trabajo, acceso a nuevos mercados, nuevas plantas industriales, y acuerdos de cooperación comercial (respuestas de I a 5).

- Pregunta 5 (a/b). Objetivos y cumplimiento de explotación industrial de resultados. Una variable importante son los objetivos finales que se planteaba el proyecto de I+D en la explotación comercial y cumplimiento del desarrollo. Esta pregunta trata de indagar los objetivos finales del proyecto como explotación industrial, explotación interna comercialización externa, explotación conjunta (respuestas de I a 5).

- Pregunta 6. Mercados de comercialización de resultados. El área donde se han comercializado los resultados, bien en mercado nacional, europeo, latinoamericano, norteamericano u otros. La respuesta es dicotómica y porcentual. 
Tabla I

Tipo de participación

\begin{tabular}{|l|c|c|c|c|c|}
\hline \multirow{2}{*}{} & \multicolumn{5}{|c|}{ Participación } \\
\cline { 2 - 6 } & Individual & $\%$ & Cooperativa & $\%$ & N \\
\hline Universidades Centros de I+D & 42 & 48,3 & 45 & 52,7 & 87 \\
Empresas & 119 & 86,9 & 18 & 13,1 & 137 \\
\hline
\end{tabular}

- Pregunta 7. Mecanismos de transferencia de tecnología. Identificar los mecanismos de transferencia tecnológica, bien a través de patentes, acuerdos de cesión tecnológica, creación de nuevas empresas, alianzas tecnológicas, licencias de producción, acuerdos de comercialización (variable dicotómica).

- Pregunta 8. Razones de participación. Se preguntan las razones de participar en un proyecto apoyado por un programa público de I+D: acceso a financiación, acceso a nuevos mercados, acceso a nuevos conocimientos, compartir costes, disminuir riesgos, prestigio o imagen (respuestas de I a 5).

- Pregunta 9. Obstáculos. Identificar los obstáculos encontrados por los participantes en el desarrollo del proyecto: dificultades técnicas, cambios en los mercados, retirada de socios, divergencia entre socios, problemas de comunicación, gestión de consorcio, falta de financiación externa, y falta sincronización financiación (respuestas de I a 5).

\section{Resultados}

\subsection{Aspectos cooperativos}

En el caso de las universidades, un 52,7\% de los proyectos son cooperativos, mientras que en el caso de las empresas solamente lo son un 13,1\% (Tabla I). Sin embargo las universidades cooperan básicamente con otras universidades (65) y centros de investigación (19) y muy poco con empresas (9). En cuanto a las empresas, cooperaban básicamente con empresas (2I) y muy poco con universidades (9) y centros de investigación (7) (Tabla 2).

\subsection{Análisis descriptivo de las variables}

El análisis de la diferencia de medias entre ambos colectivos arroja los siguientes resultados:

Valoración del proyecto (valores 0 - I)

Las universidades valoran el proyecto fundamentalmente como investigación $(\bar{x}=0,954)$ y como desarrollo tecnológico en menor valor $(\bar{x}=0,552)$. Las empresas otorgan la calificación de investigación en un lugar bajo $(\bar{x}=0,387)$ junto a la valoración de ingeniería $(\bar{x}=0,307)$, mientras definen la acción como desarrollo tecnológico $(\bar{x}=0,883)$.

Valoración de la tecnología (valores 1-5)

La tecnología se valora también de modo diverso. Para las empresas, tanto la novedad $(\bar{x}=4,044)$ como el esfuerzo organizativo del proyecto $(\bar{x}=4,073)$ son altos, mientras el riesgo $(\bar{x}=3,445)$ es medio. Para las universidades, la novedad $(\bar{x}=4,322)$ es más relevante, no así el riesgo $(\bar{x}=3,092)$ o el esfuerzo organizativo $(\bar{x}=3,360)$.

Relevancia de los objetivos del proyecto

Es relevante la diferencia entre ambos colectivos en lo que a desarrollo de nuevos productos se refiere, empresas $(\bar{x}=3,832)$ y universidades $(\bar{x}=3,172)$, si

Tabla 2

Nivel de cooperación

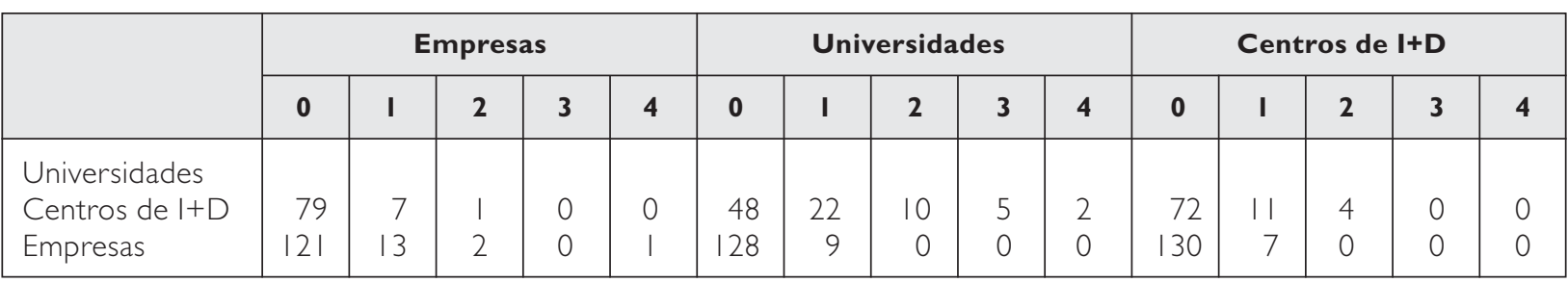


bien la diferencia no aparece significativa desde el punto de vista estadístico. Las empresas persiguen objetivos de mejora y las universidades objetivos relacionados con los demostradores (que permiten publicar), dentro de los bajos valores observados.

Cabe subrayar la poca relevancia otorgada a la mejora de procesos. La búsqueda de objetivos de búsqueda de acuerdos es baja, en general, pero sobre todo para las empresas lo que puede llamar la atención, si no consideramos que las universidades busquen alianzas en los proyectos para nuevos proyectos.

Si consideramos los objetivos en dos grupos de variables compuestas, tanto en aquellos objetivos relacionados con la mejora de conocimiento práctico (RELOBJCONOC; $\alpha=0,670$ ), como en los objetivos relativos a la innovación (RELOBJINNOV; $\alpha=0,690$ ), las medias de las empresas son superiores. $\operatorname{Sin}$ embargo, debe subrayarse que en la variable RCONOC (adquisición de conocimientos) se aprecia una diferencia muy superior en las universidades $(\bar{x}=4,287)$ frente a las empresas $(\bar{x}=3,883)$, diferencia significativa $(p<0,001)$ estadísticamente.

\section{Cumplimiento de los objetivos del proyecto}

Si se analiza el cumplimiento de objetivos genéricos, los encuestados se autocalifican en niveles bajos estando las medias situadas en torno a una puntuación de 3 con medidas de desviación típica altas. En general, las universidades puntúan en niveles inferiores a las empresas, excepto en lo referente a demostradores donde superan a las empresas $(\bar{x}=3,253$ frente a $\bar{x}=2,781$ ) y en adquisición de conocimientos donde superan a las empresas $(\bar{x}=4,299$ frente a $\bar{x}=$ $3,650)$. Las diferencias son significativas en todas las variables excepto en desarrollo de nuevos productos y mejora de procesos.

Si consideramos los objetivos en dos grupos de variables compuestas tanto en aquellos objetivos relacionados con la mejora de conocimiento práctico (CUMPLOBJCONOC; $\alpha=0,720$ ), como en los objetivos relativos a la innovación (CUMPLOBJINNOV; $\alpha=0,7$ |5), las medias de las empresas son superiores, lo que apunta un compromiso mayor en esa consecución de objetivos.

\section{Objetivos iniciales de explotación industrial} del proyecto

Si se analizan los objetivos iniciales de explotación industrial o comercialización, de forma congruente con lo anterior, las empresas puntúan siempre de modo superior a las universidades. La única excepción se sitúa en la explotación exterior, baja para todos los colectivos y sin significación en diferencia de medias. La variable compuesta OBJEXPLOT presenta valores superiores en las empresas frente a las universidades, también siendo significativas las diferencias.

Si se analizan el cumplimiento de objetivos de explotación, las tres variables (interna, externa y conjunta) presentan valores superiores en las empresas, aunque la diferencia de medias en la externa no es significativa. La media de la variable compuesta explotación total (CUMPLEXPLOT; $\alpha=0,695$ ) es de 6,78 I en las empresas y de 5,034 en las universidades, siendo significativa la diferencia $(p<0,001)$.

Mercados de explotación de los resultados del proyecto

La variable de explotación nacional es claramente superior en el caso de las empresas con $p<0,001$. La variable compuesta explotación con exportación es también superior en el caso de las empresas ( $\bar{x}=$ 0,978 frente a $\bar{x}=0,1$ I 5) con $p<0,05$. No obstante, los porcentajes son bajos en ambos casos. Por su parte, menos de un $10 \%$ en el caso de proyectos de universidad, la tecnología se exportó a mercados exteriores. En el caso de las empresas este porcentaje es del $50 \%$.

\section{Mecanismos de transferencia de tecnología del proyecto}

Los resultados obtenidos son más bien escasos, pues sólo 5 I del total de los proyectos (23\%) se han traducido en patentes ( $11 \%$ en el caso de universidades y $12 \%$ en el caso de empresas), no habiendo diferencia significativa entre ambos grupos. El resto de modalidades si presenta diferencias significativas entre ambos grupos, siendo las alianzas tecnológicas los más importantes, y donde la universidad aparece con un $25 \%$ de casos, mientras la empresa tiene sólo 8,75\% (Tabla 3).

\section{Razones para la participación en los proyectos}

Las razones para la participación en los proyectos son para la universidad, fundamentalmente, el acceso a la financiación de sus proyectos $(\bar{x}=4,|6|$ frente a $\bar{x}=3,489$ en empresas) y el acceso a nuevos conocimientos $(\bar{x}=4,506$ frente a $\bar{x}=3,723$ en 
Tabla 3

Mecanismos utilizados de transferencia de tecnología

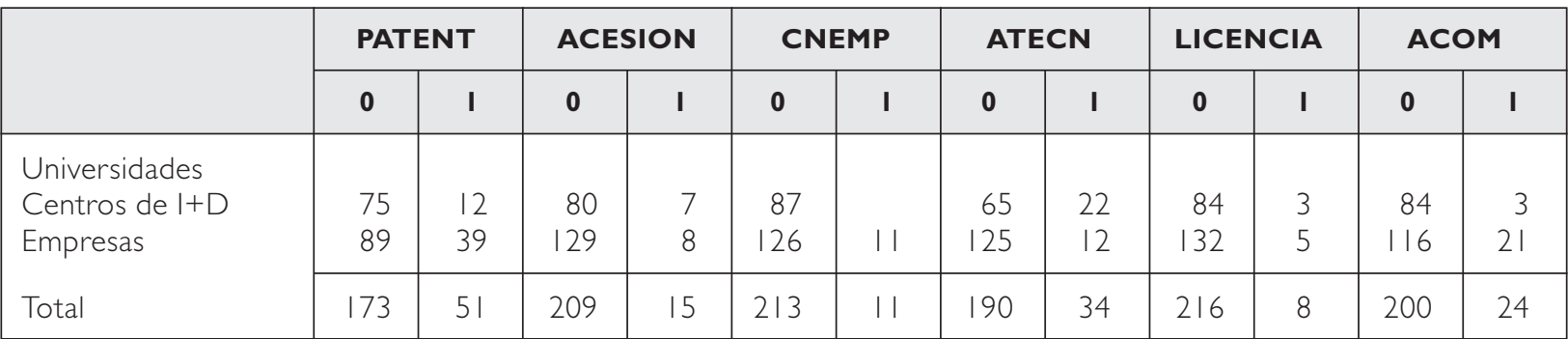

empresas). Para la empresa, el acceso a nuevos mercados ( $\bar{x}=3,453$ frente a $\bar{x}=1,322$ en universidades), las razones de prestigio e imagen $(\bar{x}=3,24$ I frente a $\bar{x}=1,770$ en universidades) y la evitación del riesgo con muy bajas puntuaciones en general ( $\bar{x}=$ 2,672 frente a $\bar{x}=1,207$ en universidades).

Barreras u obstáculos para cumplir los objetivos del proyecto

Únicamente los aspectos tecnológicos aparecen como importantes, ya que se puntúan con 3,790 de media, no existiendo diferencia significativa entre la opinión de empresas y universidades. El resto de los factores apuntados obtienen puntuaciones muy bajas, por debajo de I, lo que significa que no se consideran importantes. Sin embargo, la diferencia de medias es importante en todos ellos excepto en la financiación exterior. Las empresas señalan, a diferencia de las universidades, los cambios en el mercado como las más relevantes.

\subsection{Análisis multivariante}

Para identificar qué variables son críticas y establecer un modelo posteriormente se ha realizado un análisis factorial de ambas poblaciones: universidades-centros de I+D y empresas.

\section{Colectivo de universidades-centros de I+D}

El análisis factorial pone de relieve que la combinación de cuatro componentes explica un 80,18\% de la varianza de la muestra. Los cuatro componentes básicos son: $\mathrm{Cl}=\mathrm{f}$ (CUMPLOBCONOC, RELOB)CONOC, BARRLEGA, BARRFINANC, BAERRORG, COMPLORG); $22=f(C U M P L O B I N N O V, R E L O-$ BINNOV); $C \cdot 3=f(C U M P L E X P L O T, O B J E X P L O T) ; y$ $\mathrm{C} 4=f(\mathrm{COMPLTEC}, \mathrm{COMPLORG})$. El primero $(\mathrm{Cl})$ relacionado con el conocimiento y los obstáculos, el segundo (C2) relacionado con la relevancia y los objetivos de innovación, el tercero (C3) con los de explotación y el cuarto (C4) con la complejidad tecnológica .

Se ha desarrollado un modelo de regresión que explique el cumplimiento de los objetivos de explotación. La función resultante en esta población de universidades es la siguiente (todos con $p<0,00$ I): CUMPLEXPLOT $=$ f(cte, OBJEXPLOT, BARRLEGA, ACCONOC, RELOBCONOC, CUMPLOBJCO$\mathrm{NOC}) ; \mathrm{R} 2=0,88 \mathrm{I} ; \mathrm{p}<0,00$ I

Por tanto, las variables relevantes son los objetivos iniciales de explotación, las barreras legales, el acceso a nuevo conocimiento, la relevancia de adquisición de conocimiento y el cumplimiento de esos objetivos. Es decir, en este colectivo aparecen claramente los aspectos relativos al conocimiento como determinantes del proyecto.

\section{Colectivo de empresas}

En el análisis factorial aparecen cuatro componentes básicos cuya combinación explica un 82,53\% de la varianza de la muestra. El primero $(\mathrm{Cl})$ relacionado con el conocimiento (relevancia de objetivos relativos al mismo y cumplimiento de estos, así como con la relevancia y el cumplimiento de los objetivos de innovación). El segundo (C2) relativo al tipo de participación (individual o colectivo) y el número de empresas que cooperan. El tercero (C3) relativo a las barreras legales y de organización, y el cuarto (C4) relativo a las ventajas de compartir el riesgo. $\mathrm{Cl}=\mathrm{f}($ RELOBJCONOC, CUMPLOBCONOC, CUMPLOBINNOV, RELOBINNOV); $C 2=f(T I P O-$ PART, NEMPCOOP); $C \cdot 3=f(B A R R L E G A, \quad B A-$ RRORG); y $C 4=f(C T R I S K)$

Se ha desarrollado un modelo de regresión que explique el cumplimiento de los objetivos de explotación. La función resultante en esta población de em- 
presas es la siguiente (todos con $p<0,0001$ ): CUM-

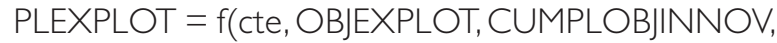
RELOBINNOV, EXPORT,TTPAT, ACING, CTERISK); $R 2=0,765 ; p<0,000$ I

Por tanto, las variables relevantes son los objetivos iniciales de explotación, la relevancia de objetivos de adquisición de conocimiento y el cumplimiento de los objetivos relativos a la innovación, la exportación de resultados, la transferencia de tecnología en patentes y licencias, las acciones de ingeniería y la Compartición del riesgo como motivos para participar. Puede observarse que, en el colectivo de empresas, aparecen claramente los aspectos relativos a la innovación y la explotación y transferencia de resultados como determinantes del proyecto.

\section{Conclusiones}

A pesar de que un importante porcentaje de los proyectos son cooperativos, las respuestas apuntan que los participantes no tienen un sentimiento de participación en este sentido. La valoración mutua del rol de universidades y empresas es muy baja, lo que indica que la distancia entre ambos colectivos es importante y supone una fuerte barrera al desarrollo tecnológico. En este sentido, las universidades y empresas entrevistadas apuntaron que en estos programas no se valora la cooperación como elemento decisorio en la concesión de la ayuda correspondiente.

Las universidades valoran el proyecto fundamentalmente como investigación y como desarrollo tecnológico en menor grado. Sin embargo, la valoración de las empresas es opuesta, complementándose con una valoración de ingeniería importante. Se recalcan otras diferencias entre ambos colectivos: para las empresas es relativamente importante el desarrollo de nuevos productos y para las universidades no. Las empresas persiguen objetivos de mejora y las universidades objetivos relacionados con los demostradores (que permiten publicar). Cabe subrayar la poca relevancia otorgada a la mejora de procesos y aparece la resistencia a la cooperación.

La propia auto evaluación de los encuestados apunta a un nivel bajo de cumplimiento de los objetivos de los proyectos, aunque las calificaciones medias de las empresas son superiores, lo que apunta un cierto compromiso mayor en esa consecución de objetivos. Si se analizan los objetivos iniciales de explotación industrial o comercialización, de forma congruente con lo anterior, las empresas puntúan siempre de modo superior a las universidades. No hay una vocación exportadora tecnológica.

Los resultados son más bien magros en cuanto a la utilización de mecanismos de transferencia tecnológica. Sólo 5 I de los proyectos (23\%) se han traducido en patentes ( 1 1\% en el caso de universidades y $12 \%$ en el caso de empresas), no habiendo diferencia significativa entre ambos grupos. En relación a las razones para la participación en los proyectos, la universidad valora principalmente el acceso a la financiación de sus proyectos y el acceso a la adquisición de nuevos conocimientos. Por su parte, la empresa valora en mayor medida el acceso a nuevos mercados y las razones de prestigio e imagen.

Al considerar las barreras u obstáculos para cumplir los objetivos del proyecto se puede concluir que únicamente los aspectos tecnológicos aparecen como importantes y no hay diferencia significativa entre la opinión de empresas y universidades. El resto de los factores apuntados obtienen puntuaciones muy bajas.

\section{Referencias bibliográficas}

ADAMS, J. D.; CHIANG, E. P., y STARKEY, K. (200I). Industry-University Cooperative Research Centers, Journal of Technology Transfer, 26, 73-86.

ANDERSON, T. R. (2007). Measuring the efficiency of university technology transfer, Technovation, 27, 5, pp. 306-318

AUTIO, E., y LAANANEN, T ( 1995). Measurement and evaluation of technology transfer: review of technology transfer mechanisms and indicators, International Journal of Technology Management, Vol. I0, Issues 7-8. pp. 643.

BERCOVITZ, J. E. L., y FELDMANN, M. P. (2006). Entrepreneurial Universities and Technology Transfer: A Conceptual Framework for Understanding Knowledge-Based Economic Development. Journal of Technology Transfer, 31, I75- I88.

BERCOVITZ, J. E. L.; FELDMAN, M. P.; FELLER, I., y BURTON, R. M. (200I). Organizational Structure as a Determinant of Academic Patent and Licensing Behaviour: An Exploratory Study of Duke, Johns Hopkins, and Pennsylvania State Universities. Journal of Technology Transfer, 26, 2 I-35.

BOZEMAN, B. (2000). «Technology Transfer and public policy: a review of research and theory», Research Policy, 29, pp. 627-655.

BRASNCOMB, L. M. (1993). The national technology policy debate, en Branscomb, M. (ed.) Empowering Technology (pp. I-35), The MIT press, Cambridge. MA. 
CALOGHIROU,Y.; TSAKANIKAS, A.; V. NICHOLAS S., y VONORTAS, N. S. (200 I ). University-Industry Cooperation in the Context of the European Framework Programmes, Journal of Technology Transfer, 26, pp. |53-|6|.

COOKE, P., y LEYDESDORFF, L. (2006). Regional Development in the Knowledge-Based Economy:The Construction of Advantage, Journal of Technology Transfer, 31: pp. 5-I 5.

COLYVAS, J.; CROW, M.; GELIJNS, A.; MAZZOLENI, R.; NELSON, R.; ROSENBERG, N., y SAMPAT, B. (2002). How Do University Inventions Get Into Practice?, Management Science, 48, I, pp. 6I-72

CHAPPLE,W.; LOCKETT, A.; SIEGEL, D., y WRIGHT, M. (2005). Assessing the relative performance of U.K. university technology transfer offices: parametric and non-parametric evidence, Research Policy, 34, pp. 369-384.

ETZKOWITZ, H.;WEBSTER, A.; GEBHARDT, C.; REGINA, B., y TERRA, C. (2000). The future of the university and the university of the future: evolution of ivory tower to entrepreneurial paradigm. Research Policy, 29, 313-330.

ETZKOWITZ, H. (2004). The evolution of the entrepreneurial university. International Journal ofTechnology and Globalisation, I, pp. 64-77.

ETZKOWITZ, H., y KLOFSTEN, M. (2005). The innovating region: toward a theory of knowledgebased regional development, $R \& D$ Management. 35, pp. 243-255.

FUNDACIÓN CYD (2006). La contribución de las universidades españolas al desarrollo, Barcelona.

GEISLER, E., y RUBENSTEIN, A. H. ( 1989). University industry relations: A review of major issues. In Link, A.N. and Tassey, G., eds. Cooperative research and development:The industry-government relationship (pp. 43-62) Boston. Luwer Academic Publishers.

GORSCHEK,T.;WOHLIN, C.; GARRE, P., Y LARSSON, S.A. (2006). A Model for Technology Transfer in Practice, IEEE Software, 23, 6, pp. 88-95, IEEE.

GREINER, M. A., y FRANZA, R. M. (2003). Barriers and Bridges for Successful Environmental Technology Transfer, Journal of Technology Transfer, 28, 2, pp. 167-177.

GUNASEKARA, C. (2006). Reframing the Role of Universities in the Development of Regional Innovation Systems. Journal of Technology Transfer, 31, IOI- II 3 .

HALL, B. H.; LINK, A. N., y SCOTT,J.T. (200 I). Barriers Inhibiting Industry from Partnering with Universities: Evidence from the Advanced Technology Program, Journal of Technology Transfer, 26, pp. 87-98.

HANNAN, M.T., y FREEMAN, J. ( 1984). Structural inertia and organizational change, American Sociological Review, 49, pp. I49- 164.
HARMON, B.; ARDISHVILI, A.; CARDOZO, R.; ELDER, T.; LEUTHOLD,T.; PARSHALL, J.; RAGHIAN, M., y SMITH, D. (1997). Mapping the university technology transfer process, Journal of Business Venturing, 12, 6, pp. 423-434.

LANE, J. P. ( 1 999). Understanding technology transfer, Assistive Technology, I I, I, pp. 5- 19

LEE, Y. S. (1996). Technology Transfer' and the Research University: A Search for the boundaries of University-Industry collaboration, Research Policy, 25, 6, pp. 843-863.

LEE, Y. S. (2002). Technology Transfer and public policy in an age of global economic competition, Policy Studies Journal, 22-2, pp. 260-266

LINK, A. N., y SCOTT, J.T. (200 I). Barriers Inhibiting Industry from Partnering with Universities: Evidence from the Advanced Technology Program, Journal of Technology Transfer, 26, 87-98

MATKIN, G.W. (1990). Technology Transfer and the University, Macmillan Publishing Company, New York.

MAZZOLENI, R. (2006). The Effects of University Patenting and Licensing on Downstream R\&D Investment and Social Welfare, Journal of Technology Transfer, 31, pp. 431-44I.

MESERI, O., y MAITAL, S. (200 I). A Survey Analysis of University-Technology Transfer in Israel: Evaluation of Projects and Determinants of Success, Journal of Technology Transfer, 26, pp. I I 5- 126.

MEYER, M. (2006). Academic Inventiveness and Entrepreneurship: On the Importance of Start-up Companies in Commercializing Academic Patents, Journal of Technology Transfer, 31, pp. 50 I-5 I0,

MORGAN, R. P.; KRUYTBOSCH C., y KANNANKUTTY, N. (200 I). Patenting and Invention Activity of U.S. Scientists and Engineers in the Academic Sector: Comparisons with Industry, Journal of Technology Transfer, 26, pp. 173-183.

MOWERY, D. C., y SHANE, S. (2002). Introduction to the Special Issue on University Entrepreneurship and Technology Transfer, Management Science, 48, I, pp. 5-9.

MOWERY, D. C., y SAMPAT, B. N. (2005). Universities in National Innovation Systems. En:

REISMAN,A. (2005). Transfer of technologies: a crossdisciplinary taxonomy, Omega, 33, pp. I 89-202.

O'SHEA R. P; ALLEN, T. J:; CHEVALIER, A., y ROCHE, F. (2005). Entrepreneurial orientation, technology transfer and spinoff performance of U.S. universities, Research Policy, 34, pp. 994- 1009.

ROGERS, E. M.; TAKEGAMI, S., y YIN, J. (200 I). Lessons learned about technology transfer, Technovation 21, pp. 253-26I.

RONDE, P., y HUSSLER, C. (2005). Innovation in regions: What does really matter?, Research Policy, 34 (2005) I I 50- I I72 
SANTORO, M. D., y GOPALAKRISHNAN, S. (200 I). Relationship Dynamics between University Research Centers and Industrial Firms:Their Impact on Technology Transfer Activities, Journal of Technology Transfer, 26, pp. |63-171.

SCHMIEMANN, M., y DURVY, J. M. (2003). New Approaches to Technology Transfer from Publicly Funded Research, Journal of Technology Transfer, 28, pp. 9-15.

SHANE, S. (200I). Technology Opportunities and New Firm Creation, Management Science, Vol. 47, 2, pp. 205-220.

SHANE, S. (2002). University technology transfer to entrepreneurial companies, Journal of Business Venturing, 17, 6, I, pp. 537-552.

SIEGEL, D. S.; WALDMAN, D., y LINK, A. S. (2003). Assessing the impact of organizational practices on the relative productivity of university technology transfer offices: an exploratory study, Research Policy 32, pp. 27-48.

SIEGEL D. S.; WALDMAN, D. A., y ATWATER, L. E. (2004). Towards a model of the effective transfer of scientific knowledge from academicians to practitioners: qualitative evidence from the commercialization of university technologies, J. Eng. Technol. Management. 21, pp. II5-1 42.
SINGH, K. (1997). The impact of technological complexity and interfirm cooperation on business survival, Academy of Management Journal, 40, 2, 339367.

STEPHAN, P. (200 I). Educational Implications of University-Industry Technology Transfer, Journal of Technology Transfer, 26, 3, pp. 199-205.

STOCK, G. N., y TATIKONDA, M. V. (2000). A Typology of Project-Level Technology Transfer Processes. Journal of Operations Management, 18, 6, pp. 719-737.

TATIKONDA, M. V., y STOCK, G. N. (2003). Product Technology Transfer in the Upstream Supply Chain, J Prod. Innov. Manag., 20, pp. 444-467.

THURSBY,J. G.; R. JENSEN., y THURSBY, M. C. (200 I). Objectives, Characteristics and Outcomes of University Licensing: A Survey of Major U.S. Universities, Journal of Technology Transfer, 26: I, 59-72.

WALKER, A., y ELLIS, H. (2000). Technology transfer: strategy, management, process and inhibiting factors. A study relating to the technology transfer of intelligent systems, International Journal of Innovation Management, 4, I, pp. 97- 122.

WILLIAMS, F., y GIBSON, D. V. (1990). Technology Transfer: A Communication Perspective, Sage Publications. 